Journal Club

Editor's Note: These short, critical reviews of recent papers in the Journal, written exclusively by graduate students or postdoctoral fellows, are intended to summarize the important findings of the paper and provide additional insight and commentary. For more information on the format and purpose of the Journal Club, please see http://www.jneurosci.org/misc/ifa_features.shtml.

\title{
A Fragile Balance at Synapses: New Evidence Supporting a Role for FMRP in Homeostatic Plasticity
}

\author{
Fredrick E. Henry \\ Neuroscience Graduate Program, University of Michigan, Ann Arbor, Michigan 48109 \\ Review of Soden and Chen
}

Homeostatic synaptic scaling operates in tandem with forms of neuronal plasticity such as long-term potentiation and long-term depression to maintain neural circuit activity within a stable range. Experimentally, homeostatic adaptations at synapses are usually induced by prolonged spike blockade [with tetrodotoxin (TTX)], yielding a form of synaptic scaling thought to involve cell-wide, transcription-dependent changes that affect every synapse on a given neuron (Turrigiano, 2008). However, more recent evidence has also identified locally mediated adaptive mechanisms that allow spatially discrete synaptic compensation within a neuron's highly compartmentalized dendritic arbor (Sutton et al., 2006; Rabinowitch and Segev, 2008). Early work in this area demonstrated that concurrent action potential and NMDA receptor (NMDAR) blockade (TTX + the NMDAR antagonist APV) promotes dendritic translation of GluA1 AMPAR subunits, leading to localized enhancement of synaptic strength (Sutton et al., 2006). Other work demonstrated that TTX and APV treatment drives intracellular synthesis of retinoic acid (RA), which reduces

Received Feb. 15, 2011; revised March 22, 2011; accepted March 23, 2011. F.E.H. was supported by a Ruth L. Kirschtein National Research Service Award. I thank Dr. Michael Sutton for helpful discussion on the manuscript.

Correspondence should be addressed to Fredrick E. Henry, Molecular and Behavioral Neuroscience Institute, University of Michigan, 109 Zina Pitcher Place, 5860 BSRB, Ann Arbor, MI 48109. E-mail: fehenry@ umich.edu.

DOI:10.1523/JNEUROSCI.0829-11.2011

Copyright $\odot 2011$ the authors $\quad 0270-6474 / 11 / 316617-03 \$ 15.00 / 0$ the association of the RNA-binding protein $\operatorname{RAR} \alpha$ with select mRNAs such as GluA1, thereby removing their translational block (Aoto et al., 2008).

Another RNA-binding protein that regulates dendritic translation is the fragile $\mathrm{X}$ mental retardation protein (FMRP) (Zalfa et al., 2003; Muddashetty et al., 2007). As evidenced by the devastating symptoms in fragile X syndrome (FXS), mutations in the Fmrl gene result in severe disruptions in cognitive and social abilities, suggesting a pivotal role for FMRP in basic learning and memory processes (Bassell and Warren, 2008). In a recent article published in The Journal of Neuroscience, Soden and Chen (2010) demonstrate that FMRP is directly involved in homeostatic plasticity, and provide evidence to support it acting within dendrites to regulate the translation of specific mRNAs in response to increases in RA signaling.

To investigate a potential role for FMRP in local synaptic scaling, Soden and Chen first examined the effects of prolonged action potential and NMDAR blockade (36 h TTX + APV) in organotypic hippocampal slices prepared from wild-type (WT) or Fmr1 knock-out (KO) mice. Whereas CA1 pyramidal neurons exhibited enhanced mEPSC amplitude following TTX + APV treatment, no such scaling response was evident in CA1 neurons from Fmr1 KO mice (their Fig. 1). Because this group had previously shown a role for RA in homeostatic scaling induced by TTX + APV treatment (Aoto et al., 2008), the authors next examined whether FMRP was required upstream or downstream of activity-dependent RA synthesis. Using a fluorescent reporter in which three tandem retinoic acid response elements (RAREs) control GFP transcription, the authors found that cultured WT and Fmr1 KO hippocampal neurons displayed equivalent levels of RARE-GFP reporter expression after TTX+APV treatment (their Fig. 2). However, the protein synthesis-dependent increase in mEPSC amplitude induced by direct RA treatment ( $4 \mathrm{~h}$ ) was lost in Fmrl KO neurons. In addition, Soden and Chen found that RA application induced a translation-dependent increase in the levels of GluA1, GluA2, and eEF2 in synaptoneurosomes in organotypic slices from WT, but not Fmr1 KO, slices (their Fig. 5). Finally, in studies where specific proteins were immunoprecipitated following metabolic labeling with radiolabeled amino acids, the increase in GluA1 synthesis driven by RA treatment was also lost in Fmrl KO neurons. Together, these results suggest that FMRP plays a role downstream of RA in dendritic protein synthesis, where it is required for the translation of specific mRNAs in response to RA signaling.

Although Soden and Chen (2010) demonstrated a critical role for FMRP in homeostatic plasticity dependent on RA synthesis, interestingly, they found that neither FMRP nor RA was required for homeostatic enhancement of synaptic strength induced by TTX alone [Soden and Chen (2010), their Fig. 4]. In WT 
and Fmr1 KO neurons, $60 \mathrm{~h}$ of TTX treatment induced a similar increase in mEPSC amplitude, and in each case the enhanced mEPSCs were resistant to inhibition with the polyamine philanthotoxin, which targets GluA2-lacking AMPARs. In contrast, scaled mEPSCs following TTX+ APV treatment are specifically reversed by polyamine toxins [Sutton et al. (2006); Aoto et al. (2008); Soden and Chen (2010), their Fig. 2]. These observations underscore the notion that multiple forms of homeostatic plasticity exist in hippocampal neurons, and suggest that FMRP plays a specific role in synaptic adaptations that require local translation near synaptic sites.

Rescue experiments using viral expression of FMRP in organotypic hippocampal slices from Fmr1 KO mice indicated that proper RA-induced scaling in CA1 neurons can be restored by returning FMRP to appropriate levels (their Fig. 7). That acute restoration of FMRP expression can restore RA-induced scaling argues against the possibility that defects in synapse development or maturation in Fmr1 KO neurons accounts for the absence of homeostatic control, and supports a specific requirement for FMRP in RA-induced scaling. To elucidate FMRP's mechanism of action in RA-induced scaling, Soden and Chen assessed the efficacy of two FMRP mutants in additional rescue experiments. One mutant, FMRP $\Delta$ RGG, lacked the RGG box necessary for binding to G-quartet structures on select target mRNAs. The other mutant, FMRP(I304N), carried a point mutation in a crucial $\mathrm{KH}$ domain necessary for binding to mRNAs with a "kissing complex" motif. Neither of these mutants had an effect on AMPAR levels or basal synaptic transmission when expressed in WT neurons (their supplemental Fig. 5). Likewise, expression of FMRP $\Delta$ RGG-GFP in slices from Fmr1 KO mice had no effect on baseline mEPSC amplitude (their Fig. 7) or GluA1 levels as assessed via Western blotting (their Fig. 8). In contrast, expression of FMRP(I304N)-GFP decreased basal mESPC amplitude and reduced GluA1 protein levels in Fmr1 KO cells (their Fig. 7, Fig. 8). Given previous work showing an interaction between FMRP and GluA1 mRNA in synaptoneurosomes (Muddashetty et al., 2007), these results raise the possibility that FMRP may normally associate with and suppress the translation of GluA1 mRNA via its RGG box and not its KH domain, though Soden and Chen did not directly examine the mRNA binding properties of either mutant. Importantly, neither mutant rescued scaling induced by RA or TTX+APV treatment, indicating that recovery of FMRP's ability to suppress GluA1 synthesis may not be sufficient to restore its role in RA-induced dendritic translation.

The differential effect of the FMRP mutants on baseline mEPSC amplitude and GluA1 levels suggests that their inability to rescue synaptic scaling results from distinct impairments. The inability of FMRP $\Delta$ RGG to rescue synaptic scaling in Fmr1 KO cells may stem from the possibility that the RGG box is necessary for FMRP's interaction with GluA1 mRNA. If this is the case, why then is expression of the FMRP(I304N) mutant, which contains an intact RGG box and clearly exerts a suppressive effect on GluA1 expression, not successful in restoring RA-induced scaling? Previous reports have shown that this mutation blocks the ability of FMRP to associate with translating polyribosomes, as well as with its autosomal paralog FXR2P (Feng et al., 1997). It may be that mRNP particles containing FMRP must be associated with actively translating ribosomes for RA-induced translational regulation to occur, so disrupting this interaction renders $\mathrm{FMRP}(\mathrm{I} 304 \mathrm{~N})$ unable to rescue the scaling effect in Fmr1 $\mathrm{KO}$ cells. On the other hand, given recent data suggesting that FMRP and FXR2P play unique roles during the expression of mGluR-LTD (Zhang et al., 2009), it is possible that FXR2P may also serve an important function during RA-induced synaptic scaling, and that this function requires its interaction with FMRP. Future experiments should clarify the precise aspect of FMRP function that is necessary for homeostatic plasticity.

In the experiments detailed above, Soden and Chen have provided compelling evidence that FMRP plays an important role in RA-induced synaptic scaling. However, one remaining unanswered issue concerns the mechanism by which FMRP regulates the effects of RA on dendritic protein synthesis. In coimmunoprecipitation studies, Soden and Chen found no evidence for a direct physical interaction between FMRP and RAR $\alpha$ (their Fig. 6), yet FMRP was clearly required for increased translation signaled through $\operatorname{RAR} \alpha$. The authors suggest that FMRP and RAR $\alpha$ need to be bound to the same mRNAs for RA-induced translational changes to occur. Indeed, two of the RAR $\alpha$ targets highlighted in this study, GluA1 and GluA2 mRNA, are also known targets of FMRP (Muddashetty et al., 2007), suggesting that combinatorial action of these two RNA-binding proteins might be responsible for mediating the specific alterations in translational efficiency necessary for local homeostatic plasticity. While this idea is attractive, it is also possible that FMRP is necessary for the appropriate targeting of GluA1 mRNA to specific RA-response sites near synapses. Another possibility is that FMRP, through an allosteric mechanism, operates in concert with RA to induce the conformational changes in $\operatorname{RAR} \alpha$ necessary for translational disinhibition of specific mRNAs. In the Chen laboratory's initial series of studies of RAinduced synaptic scaling, they found that treatment with the RAR $\alpha$-specific agonist AM580 was sufficient to stimulate local synthesis of GluA1 and cause an enhancement of mEPSC amplitude (Aoto et al., 2008). Although the authors clearly demonstrate that scaling induced by direct RA application is lost in FMR1 KO neurons, it would be informative to know whether this same effect is observed following RAR $\alpha$ activation with AM580.

Although a number of important questions remain, Soden and Chen's work clearly establishes a role for FMRP in homeostatic control of excitatory synapse function and serves to further our understanding of the synaptic etiology of FXS. To this point, the abnormal mGluR-LTD observed in FMR1 KO mice has received considerable attention as a potential link to the cognitive dysfunction associated with this disorder. These new results raise the intriguing possibility that dysfunctional homeostatic regulation, perhaps in concert with defects in mGluR-dependent signaling in neurons, plays an important role in the devastating symptoms of FXS. The extent to which altered homeostatic plasticity contributes to the pathogenesis of FXS and other such disorders is an important area of future study.

\section{References}

Aoto J, Nam CI, Poon MM, Ting P, Chen L (2008) Synaptic signaling by all-trans retinoic acid in homeostatic synaptic plasticity. Neuron 60:308-320.

Bassell GJ, Warren ST (2008) Fragile X syndrome: loss of local mRNA regulation alters synaptic development and function. Neuron 60:201-214

Feng Y, Absher D, Eberhart DE, Brown V, Malter HE, Warren ST (1997) FMRP associates with polyribosomes as an mRNP, and the I304N mutation of severe fragile X syndrome abolishes this association. Mol Cell 1:109-118.

Muddashetty RS, Kelić S, Gross C, Xu M, Bassell GJ (2007) Dysregulated metabotropic glutamate receptor-dependent translation of AMPA receptor and postsynaptic density-95 mRNAs at synapses in a mouse model of 
fragile X syndrome. J Neurosci 27:53385348.

Rabinowitch I, Segev I (2008) Two opposing plasticity mechanisms pulling a single synapse. Trends Neurosci 31:377-383.

Soden ME, Chen L (2010) Fragile X protein FMRP is required for homeostatic plasticity and regulation of synaptic strength by retinoic acid. J Neurosci 30:16910-16921.
Sutton MA, Ito HT, Cressy P, Kempf C, Woo JC, Schuman EM (2006) Miniature neurotransmission stabilizes synaptic function via tonic suppression of local dendritic protein synthesis. Cell 125:785-799.

Turrigiano GG (2008) The self-tuning neuron: synaptic scaling of excitatory synapses. Cell 135:422-435.

Zalfa F, Giorgi M, Primerano B, Moro A, Di
Penta A, Reis S, Oostra B, Bagni C (2003) The fragile $\mathrm{X}$ syndrome protein FMRP associates with BC1 RNA and regulates the translation of specific mRNAs at synapses. Cell 112:317-327.

Zhang J, Hou L, Klann E, Nelson DL (2009) Altered hippocampal synaptic plasticity in the FMR1 gene family knockout mouse models. J Neurophysiol 101:2572-2580. 